\section{TRANSCRIPTOMIC ANALYSIS OF MELANOMA PATIENTS IN ADJUVANT SETTING TREATED WITH ANTI PD1 THERAPY: REAL LIFE STUDY}

${ }^{1}$ Domenico Mallardo*, 'Claudia Trojaniello, 'Maria Grazia Vitale, 'grazia d'angelo, ${ }^{2}$ Andrew White, ${ }^{1}$ Mariaelena Capone, ${ }^{1}$ Antonio Sorrentino, ${ }^{1}$ Gabriele Madonna, ${ }^{1}$ Marilena Tuffanelli, ${ }^{1}$ Vito Vanella, ${ }^{1}$ Lucia Festino, ${ }^{1}$ Ester Simeone, ${ }^{1}$ Corrado Caracò ${ }^{1}$ Nicola Normanno, ${ }^{2}$ Sarah Warren, ${ }^{1}$ Paolo Ascierto. 'Istituto Nazionale Tumori IRCCS Pascale, Naples, Italy; ${ }^{2}$ NanoString Technologies, Seattle, WA, USA

Background Adjuvant treatment of melanoma patients with immune-checkpoint inhibition (ICI) significantly improved relapse-free survival (RFS). ${ }^{1}$ In the phase 3 keynote-054 trial showed that pembrolizumab (anti-PD1) administration in adjuvant setting provided a longer RFS $(59,8 \%)$ than the placebo group $(41,4 \%)$ at a 3.5 -year median follow-up. ${ }^{2}$ Moreover, 4 years RFS results from the phase 3 checkmate 238 trial, showed a superior efficacy of nivolumab versus ipilimumab in patients with resected AJCC-7 stage III or IV melanoma. RFS rate was of $58 \%$ in the nivolumab arm and $45 \%$ in the ipilimumab arm. ${ }^{3}$ Although treatment with ICIs has improved the RFS of melanoma patients in adjuvant setting, there is still a large proportion of patients who do not respond to the treatment and then relapse. The aim of this study was to investigate the molecular mechanisms underlying resistance to antiPD1 treatment in the adjuvant setting.

Methods From December 2018 to July 2020, n. 121 melanoma patients in stage III or IV NED were treated with antiPD1s as adjuvant (minimum follow up of 12 months, range 12-30 months). These patients received nivolumab $(\mathrm{n}=95)$ or pembrolizumab $(\mathrm{n}=26)$. Distant and local metastases was observed in $33(27 \%)$ and 7 (6\%) patients, respectively (patients baseline characteristics are listed in table1). Gene expression profiles, using NanoString IO 360 panel, were performed from peripheral blood mononuclear cell (PBMCs), collected retrospectively, from n.73 patients (of which n.26 had relapse). All patients have appropriately signed informed consent. Statistical analysis was performed via Bonferroni correction, $\mathrm{P}<0.05$ was considered statistically significant for median stratification.

Results At a minimum follow-up of 12 months, the 12-month rate of Relapse-free survival was $72 \%$, confirming the data reported by checkmate 238 trial. In the transcriptomic analysis we observed that in patients with local-regional metastases there was a higher expression of ITGA2 $(\mathrm{p}<0.05)$, a gene that promotes malignant tumor aggression by up-regulating PD-L1 expression through STAT3 pathway and the downregulation of DUSP1 $(\mathrm{p}<0.05)$ that is linked in promotion of angiogenesis, invasion and metastasis. Moreover, in male group we found a higher expression of HLA-DQB1 and HLA-DQA1 which belonged to HLA class II beta chains.

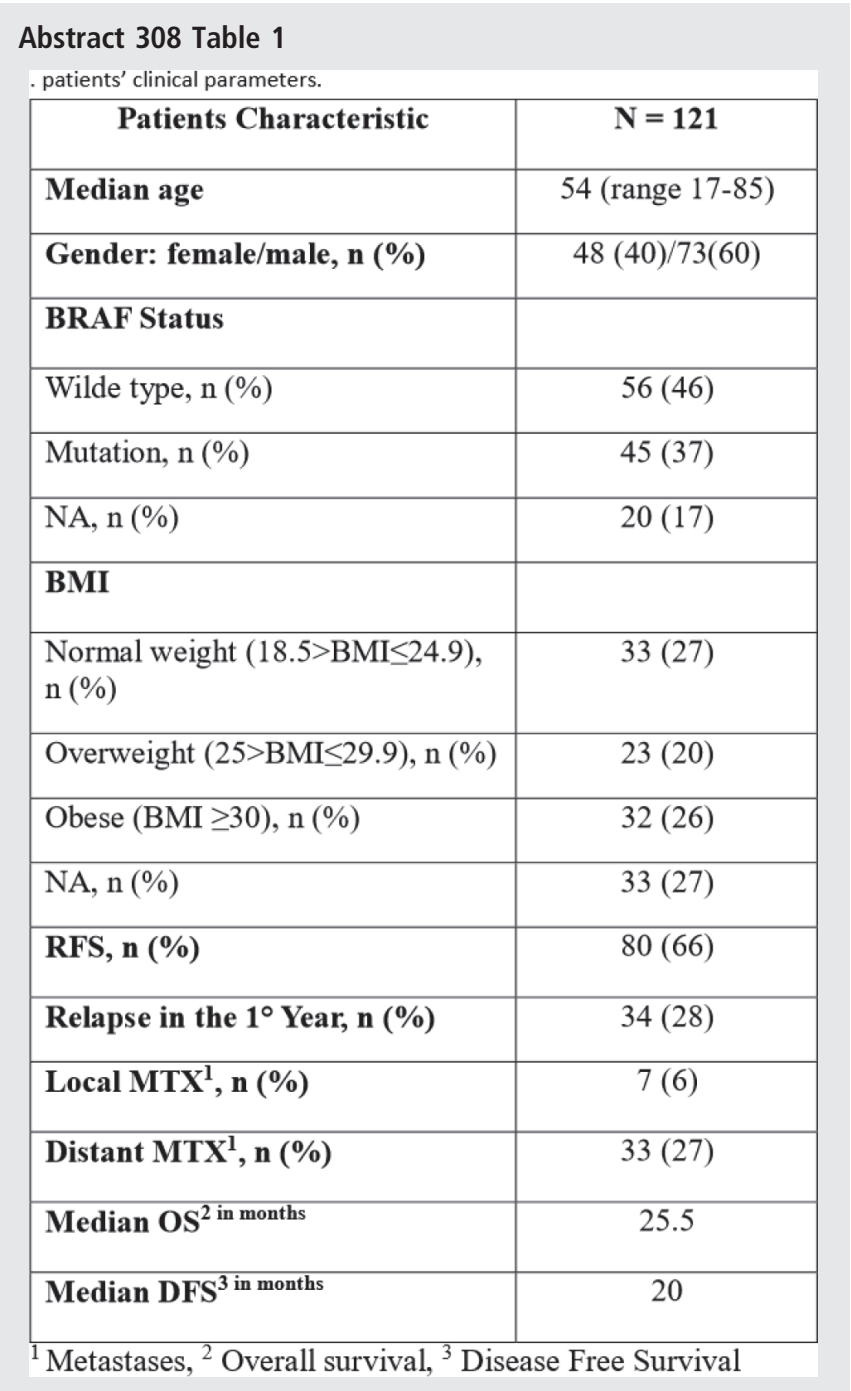

Conclusions In this preliminary report we found that RFS 1yr rate is similar to checkmate 238 study, and that patients with local metastasis have a higher expression of genes related to promote PDL1 levels. Further investigations are needed to get additional information.

Acknowledgements The study was supported by the Institutional Project "Ricerca Corrente" of Istituto Nazionale Tumori IRCCS Fondazione "G. Pascale" of Napoli, Italy.

\section{REFERENCES}

1.. Weber J, Mandala M, Del Vecchio M, et al, CheckMate 238 Collaborators. Adjuvant nivolumab versus ipilimumab in resected stage III or IV melanoma. N Engl J Med 2017 November 9:377(19):1824-1835. 
2. . Eggermont AMM, Blank CU, Mandalà M, et al. EORTC melanoma group. Adjuvant pembrolizumab versus placebo in resected stage III melanoma (EORTC 1325MG/KEYNOTE-054): distant metastasis-free survival results from a double-blind, randomised, controlled, phase 3 trial. Lancet Oncol 2021 May;22(5):643-654.

3. . Ascierto PA, Del Vecchio M, Mandalá $M$, et al. Adjuvant nivolumab versus ipilimumab in resected stage IIIB-C and stage IV melanoma (CheckMate 238): 4-year results from a multicentre, double-blind, randomised, controlled, phase 3 trial. Lancet Oncol 2020 November;21(11):1465-1477.

Ethics Approval The study was approved by internal ethics board of the Istituto Nazionale Tumori IRCCS Fondazione "G. Pascale" of Napoli Italy, approval number of registry 33/ 17 OSS.

Consent Written informed consent was obtained from the patient for publication of this abstract and any accompanying images. A copy of the written consent is available for review by the Editor of this journal.

http://dx.doi.org/10.1136/jitc-2021-SITC2021.308 